\title{
Top quark forward-backward asymmetry in $R$-parity violating supersymmetry
}

\author{
Grace Dupuis* and James M. Cline ${ }^{\dagger}$ \\ Department of Physics, McGill University, 3600 Rue University, Montréal, Québec, \\ Canada, H3A 2T8
}

\begin{abstract}
The interaction of bottom squark-mediated top quark pair production, occuring in the $R$-parity violating minimal supersymmetric standard model (MSSM), is proposed as an explanation of the anomalously large $t \bar{t}$ forward-backward asymmetry (FBA) observed at the Tevatron. We find that this model can give a good fit to top quark data, both the inclusive and invariant mass-dependent asymmetries, while remaining consistent (at the 2- $\sigma$ level) with the total and differential production cross-sections. The scenario is challenged by strong constraints from atomic parity violation (APV), but we point out an extra diagram for the effective down quark- $Z$ vertex, involving the same coupling constant as required for the FBA, which tends to weaken the APV constraint, and which can nullify it for reasonable values of the top squark masses and mixing angle. Large contributions to flavor-changing neutral currents can be avoided if only the third generation of sparticles is light.
\end{abstract}

\section{Introduction}

Recent analyses by both the CDF and $\mathrm{D} \emptyset$ collaborations have reported an anomalously large forward-backward asymmetry (FBA) in top pair production at the Tevatron. The observed discrepancy between the Tevatron data and the standard model (SM) prediction indicates the possibility of new physics. Within the SM, the top quark FBA is predicted to be identically zero at leading order (LO), with a nonzero asymmetry being generated at next-to-leading order (NLO), from extra gluon radiation and interference of one-loop box diagrams with the LO gluon exchange process. This predicted NLO asymmetry however, is much lower than that which is observed at Tevatron.

\footnotetext{
*dupuisg@physics.mcgill.ca

${ }^{\dagger}$ jcline@physics.mcgill.ca
} 
Reports from the CDF collaboration give inclusive asymmetries, measured in the $t \bar{t}$ centre of mass frame, of $A_{F B}^{t \bar{t}}=0.155 \pm 0.048$ [1], $A_{F B}^{t \bar{t}}=0.158 \pm 0.074$ [2], $A_{F B}^{t \bar{t}}=0.24 \pm 0.14$ [3] and $A_{F B}^{t \bar{t}}=0.42 \pm 0.15 \pm 0.05$ [4], with the last value corresponding to a semileptonic decay channel, while $\mathrm{D} \emptyset$ has reported a value of $A_{F B}^{t \bar{t}}=0.196 \pm 0.060_{-0.026}^{+0.018}[5,6]$. The results display a significant discrepancy with the NLO SM prediction of $A_{F B}^{S M}=0.0724_{-0.0067-0.0027}^{+0.0104+0.0020}$ [7]. Moreover, CDF observed a rise in the asymmetry with both the invariant top mass and top rapidity, reporting the following values [1]

$$
\begin{aligned}
& A_{\ell} \equiv A_{F B}^{t \bar{t}}\left(M_{t \bar{t}}<450 \mathrm{GeV}\right)=0.078 \pm 0.054 \\
& A_{h} \equiv A_{F B}^{t \bar{t}}\left(M_{t \bar{t}} \geq 450 \mathrm{GeV}\right)=0.296 \pm 0.067
\end{aligned}
$$

compared with the NLO SM predictions of [7]

$$
\begin{aligned}
& A_{S M}^{t \bar{t}}\left(M_{t \bar{t}}<450 \mathrm{GeV}\right)=0.052_{-0.006}^{+0.009} \\
& A_{S M}^{t \bar{t}}\left(M_{t \bar{t}} \geq 450 \mathrm{GeV}\right)=0.111_{-0.009}^{+0.017}
\end{aligned}
$$

The $\mathrm{D} \emptyset$ collaboration does not observe this same rise in the asymmetry with invariant mass, although the same excess in the inclusive asymmetry is observed [5].

Various models of new physics have been proposed to account for the FBA. They are typically classified by the nature of the new mediator particles, depending upon whether they are $t$ - or $s$-channel exchange of vector or scalar bosons. In general, $s$-channel models involve exchange of a colour-octet vector, either in chiral colour models proposing axigluonmediated top pair production [8-19], or Randall-Sundrum models and the associated effects of Kaluza-Klein gluons in warped extra dimensions [20-23], as well as general extensions and exotic representations of the SM colour gauge group [24-27]. These models have the advantage of a large interference with SM gluon exchange. Additionally, a colour-octet only requires nonzero axial couplings to produce the FBA, whereas colour-singlets require both vector and axial-vector couplings to $q \bar{q}$ and $t \bar{t}[28]$.

$t$-channel models involve the exchange of scalar or vector mediators whose couplings can either be flavor-conserving or have large flavor violation. In the latter case, the generation of a sufficient asymmetry requires a large $u-t$ or $d$ - $t$ coupling and concerns then arise regarding the generation of large flavor-changing neutral currents (FCNCs) [28]. Flavor-conserving models [29-34] are advantageous in this respect. Flavor-violating models typically include extensions of the SM electroweak gauge group, involving the exchange of new $W^{\prime}$ or $Z^{\prime}$ bosons with vector-like [35-46] or chiral [47-49] couplings.

Any new physics contributing to the FBA is significantly constrained by complementary top quark data, from both the Tevatron and the LHC, which agree with SM predictions. Such data in particular includes the total inclusive $t \bar{t}$ production cross-section, $\sigma_{t \bar{t}}$, and the $t \bar{t}$ invariant mass distribution of the differential top pair cross-section, $d \sigma / d M_{t \bar{t}}$. CDF finds a total production cross-section of $\sigma_{t \bar{t}}=(6.9 \pm 1.0) \mathrm{pb}$ at $4.6 \mathrm{fb}^{-1}$ [50], which agrees with the NLO and NNLO SM predictions. Reference [51] gives a NNLO value of $\left(\sigma_{t \bar{t}}=\right.$ $\left.6.63_{-0.40-0.24}^{+0.00+0.33}\right) \mathrm{pb}$, obtained using the MSTW2008 parton distribution function set [52]. 
Many $t$-channel scalar exchange models have been noted to produce too great a contribution to $d \sigma / d M_{t \bar{t}}$ at high invariant masses, if they are tuned to give a large FBA in this regime $[29,34,53,54]$. This contribution is of particular concern when considering the LHC data corresponding to this observable, which are more sensitive to the effect at high invariant masses. An additional LHC constraint is provided by measurements of the charge asymmetry, a quantity which is directly related to the Tevatron FBA, which show agreement with the SM prediction. A recent ATLAS result gives $A_{c}=-0.019 \pm 0.028 \pm 0.024$ [55], compared with a SM prediction of $A_{c}^{S M}=0.0115 \pm 0.0006$ [56]. Moreover other models (including the one featured in this paper, it will be seen) are challenged by constraints from FCNCs such as $B_{d}-\bar{B}_{d}$ mixing [13], and electroweak precision data.

In the present work, we focus on the $\tilde{b}_{R}^{*} \bar{t}_{R} d_{R}^{c}$ coupling of the $R$-parity violating, minimal supersymmetric standard model (MSSM) as an explanation of the top quark FBA. ${ }^{1}$ While $R$-parity conservation is a simple way to avoid catastrophic baryon violation in the MSSM such as proton decay, the $\tilde{b}_{R}^{*} \bar{t}_{R} d_{R}^{c}$ operator by itself does not lead to proton decay. Its coupling is constrained by neutron-antineutron oscillations [59], but the loop diagram giving rise to this constraint is suppressed if the Wino mass is large or if the squark mixing is small, as we shall assume in this work. Other bounds on $R$-parity interactions are on products of different couplings and can be evaded if the one of interest is dominant. Such operators also have the virtue of helping to provide a mechanism for baryogenesis [60].

Although the $R$-parity violating model falls in the category of those claimed to be disfavored because of the tension with the asymmetry at high invariant masses, we believed it had sufficiently strong theoretical motivation to suspend judgment on this issue. In the meantime, the more recent measurement of $A_{h}$ reported in [1] greatly ameliorates this tension. In agreement with $[57,58]$, we find that the $\tilde{b}$-mediated down quark-antiquark annihilation process can account for the observed value of the FBA, while remaining consistent with the constraints posed by the total and differential production cross-sections.

It was recently pointed out [61] that atomic parity violation (APV) provides an additional powerful low-energy constraint on $t$-channel models of the FBA. The same couplings of $u-t$ or $d-t$ to the new mediator that generate the FBA can generically produce large anomalous couplings of $u$ and $d$ to $Z$ that contribute to the weak charge of nuclei. We agree that for generic values of the stop mass matrix, the APV constraints from cesium rule out the regions of parameter space compatible with the FBA in the $R$-parity violating MSSM; however, if there is significant stop mixing and a large mass splitting, we show that there exists a canceling contribution to the anomalous parity violation which can greatly relax the constraints and preserve the viability of the model for explaining the top quark observations.

In section 2, we describe the computation of the FBA and in section 3 the new contribu-

\footnotetext{
${ }^{1}$ As this work was nearing completion, ref. [57] appeared, which did a similar analysis, however without considering in detail the atomic parity violation constraint. We became aware of ref. [1] by reading this paper. At the same time, the study [58] appeared, which considers essentially the same model, though without emphasizing its relation to the MSSM and also without reference to APV.
} 
tions to APV. Results of our numerical analysis are presented in section 4, and conclusions given in 5, including a brief discussion of the problems of FCNCs and perturbativity of the large $R$-parity violating coupling.

\section{Top pair production in $R$-parity violating SUSY}

The MSSM without $R$-parity is supplemented by the additional superpotential contributions

$$
\mathcal{W}_{\not K}=\frac{1}{2} \lambda_{i j k} L_{i} L_{j} E_{k}^{c}+\lambda_{i j k}^{\prime} \delta^{\alpha \beta} L_{i} Q_{j \alpha} D_{k \beta}^{c}+\frac{1}{2} \lambda_{i j k}^{\prime \prime} \epsilon^{\alpha \beta \gamma} U_{i \alpha}^{c} D_{j \beta}^{c} D_{k \gamma}^{c}+\mu_{i} L_{i} H_{2}
$$

where the chiral superfields $L_{i}$ and $Q_{i}$ represent the left-handed lepton and quark doublets, $E_{i}$ and $U_{i}, D_{i}$ are the right-handed lepton and quark singlets, and $H_{1,2}$ are the Higgses. ${ }^{2}$ For the top quark FBA however, we are only interested in the baryon-violating $\lambda^{\prime \prime}$ term, which gives rise to $d \bar{d} \rightarrow t \bar{t}$ via $\tilde{b}$ exchange, through the first of the two interactions

$$
\mathcal{L}_{\lambda^{\prime \prime}}=-\lambda_{i j k}^{\prime \prime} \epsilon_{\alpha \beta \gamma}\left[\left(\tilde{d}_{R}^{k \gamma}\right)^{\dagger} \bar{u}_{R}^{i \alpha}\left(d_{R}^{j \beta}\right)^{c}+\frac{1}{2}\left(\tilde{u}_{R}^{i \alpha}\right)^{\dagger} \bar{d}_{R}^{j \beta}\left(d_{R}^{k \gamma}\right)^{c}\right]+\text { h.c. },
$$

or more specifically (focusing on the relevant generations for the FBA and APV)

$$
\mathcal{L}_{\text {int }}=f_{d t} \epsilon^{\alpha \beta \gamma}\left(\tilde{b}_{\gamma}^{\dagger} \bar{t}_{\alpha} P_{L} d_{\beta}^{c}+\tilde{t}_{\gamma}^{\dagger} \bar{b}_{\alpha} P_{L} d_{\beta}^{c},\right)+\text { h.c. }
$$

where $P_{R, L}=\left(1 \pm \gamma_{5}\right) / 2$ denote the chirality projection operators and the coupling $-\lambda_{313}^{\prime \prime}$ is now denoted by $f_{d t}$. The corresponding Feynman diagram for the new contribution to $d \bar{d} \rightarrow t \bar{t}$ is shown in Figure 1. It involves only the first interaction in (3), while the second enters into the computation of APV observables to be discussed below.

Structurally similar models for the $t \bar{t}$ FBA have been considered more generally under the guise of scalar, diquark-mediated, $u$-channel top pair production within a framework of generalized exotic colour representations under the $\mathrm{SM}$ gauge group $\mathrm{SU}(3)_{c} \times \mathrm{SU}(2)_{L} \times$ $\mathrm{U}(1)_{Y}[62]$. Since it can be shown [63] that under the assumption of minimal flavour violation and avoiding FCNCs, a scalar octet is associated with quark masses and hence, the corresponding $s$-channel process mediated by a colour-octet is negligible [62], the cases of interest are the colour-triplet and sextet. A particular instance of the colour-triplet, specifically the representation $(3,1,-1 / 3)$, has the same quantum numbers as the bottom squark, and so corresponds to the case of interest in this work.

The interaction (3) involves only the right-handed squarks. In general, one expects mixing between the left and right squarks due to off-diagonal terms in the mass matrix. For a given flavor of squark, these terms are proportional to the corresponding quark mass,

\footnotetext{
${ }^{2}$ The superscript $c$ denotes charge conjugation and the indices $(i, j, k)$ and $(\alpha, \beta, \gamma)$ correspond respectively to generation and colour
} 

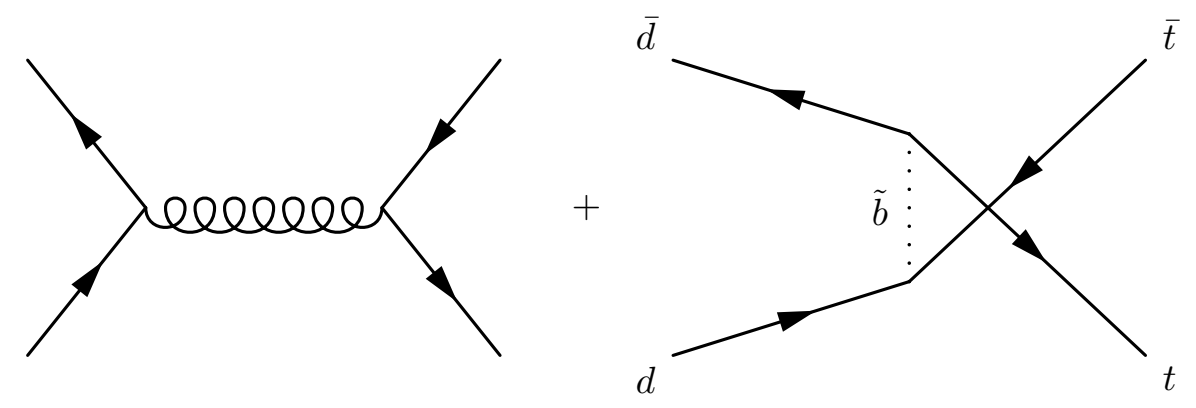

Figure 1: Feynman diagrams showing both the SM (left) and MSSM (right) contributions to the process $d \bar{d} \rightarrow t \bar{t}$.

and thus squark mixing is generally only considered for the third generation. For our FBA analysis, we neglect mixing of the bottom squark, but we will allow for top squark mixing when we discuss a loophole to the atomic parity violation constraints.

Detailed formulas for our computation of the FBA are given in appendix A.

\section{Atomic parity violation}

As mentioned in the introduction, the $\tilde{b}^{*} \bar{d}_{R}^{c} t_{R}$ coupling also affects the one-loop effective $\bar{d}_{R} \& d$ vertex and hence is subject to the constraints of atomic parity violating data. The relevant Feynman diagrams are shown in Figure 2. In the case of vanishing squark mixing, only the first diagram contributes to the anomalous coupling of the right-handed down

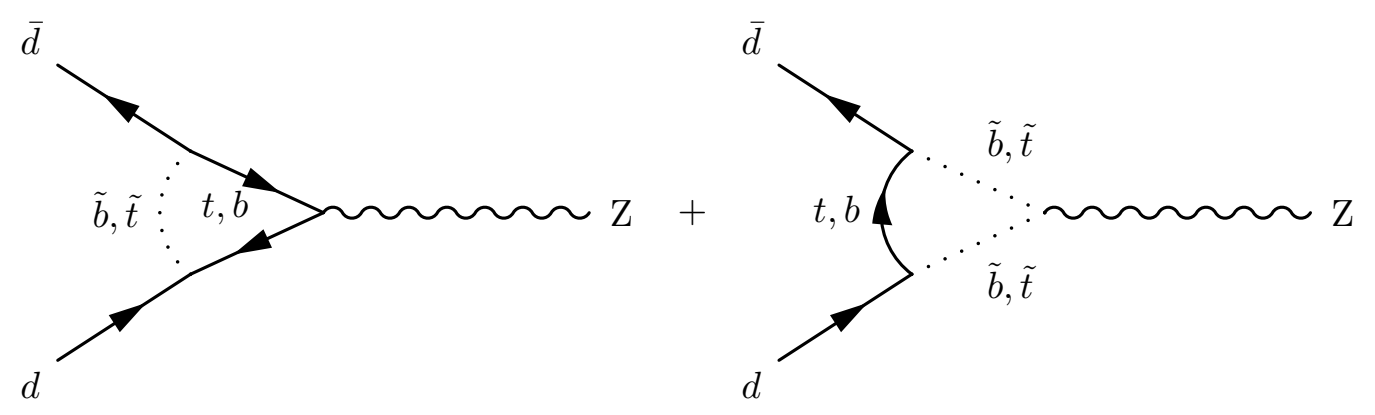

Figure 2: Anomalous coupling of the $Z$ boson to $d$ quark at one loop, arising from $\tilde{b}^{*} \bar{d}_{R}^{c} t_{R}$ and $\tilde{t}^{*} \bar{b}_{R}^{c} t_{R}$ couplings. Left: standard contribution, present with no squark mixing; right: extra contributions arising from nonzero squark mixing. Note that the diagrams with $b$ and $\tilde{t}$ in the loop arise from the second term in (3). 
quark to the $Z[61],^{3}$

$$
a_{R, d}^{N P}=\frac{f_{d t}^{2}}{16 \pi^{2}}\left(F_{1}\left(m_{t}^{2} / m_{\tilde{b}}^{2}\right)-F_{1}\left(m_{b}^{2} / m_{\tilde{t}}^{2}\right)\right)
$$

where $F_{1}$ is defined as

$$
F_{1}(x) \equiv x \frac{(x-1-\ln x)}{(1-x)^{2}} .
$$

The coefficient is normalized such that the corresponding one-loop effective Lagrangian is

$$
\mathcal{L}_{\text {eff }}=-\frac{g_{2}}{\cos \theta_{W}} a_{R, d}^{N P} Z^{\mu} \bar{d}_{R} \gamma_{\mu} d_{R}
$$

where $g_{2}$ is the $\mathrm{SU}(2)_{L}$ coupling and $\theta_{W}$ is the Weinberg angle. Since $F_{1} \sim-x \ln x$ as $x \rightarrow 0$, the second term with $x=m_{b} / m_{\tilde{t}}$ is negligible.

In the $R$-parity violating MSSM, if there is squark mixing then there are necessarily additional contributions from the right-most diagrams of fig. 2. The one with $t$ and $\tilde{b}$ in the loop has the same sign as the dominant contribution to $(4) .{ }^{4}$ Since we are interested in finding a canceling contribution, let us suppose that the $b$ squark mixing angle is small, and focus on the diagram with $b$ and $\tilde{t}$ in the loop. This has the opposite sign to (4) as desired. We define $\delta$ such that the squared stop masses are given by $m_{\tilde{t}_{1,2}}^{2}=m_{0}^{2}(1 \pm \delta / 2)$. In terms of the $\tilde{t}$ mixing angle $\theta_{\tilde{t}}$, we find that the extra contribution to $a_{R}^{N P}(d)$ is given by

$$
\delta a_{R, d}^{N P}=-\frac{f_{d t}^{2}}{32 \pi^{2}} \sin ^{2}\left(2 \theta_{\tilde{t}}\right) F_{2}(\delta)
$$

in the limit where $m_{b} \ll m_{0}$, where

$$
F_{2}(\delta)=\frac{1}{\delta} \ln \left(\frac{2+\delta}{2-\delta}\right)-1
$$

Depending upon the properties of the top squark, this extra contribution can be significant compared to the first one, $a_{R, d}^{N P}$. Suppose for example that the top squark mass splitting is large, $\delta=1.8$, corresponding to masses $m_{\tilde{t}_{1,2}}=(0.32,1.38) m_{0}$, for which $F_{2}=0.64$. A large cancellation against the first diagram can be achieved at $m_{\tilde{b}}=600 \mathrm{GeV}$ (relevant for the benchmark model we designate below), where $2 F_{1}=0.31$, if $\sin ^{2} 2 \theta_{\tilde{t}} \cong 0.5$, which is large but not maximal mixing. The weakening of the Cs APV constraint for more general values of $m_{\tilde{b}}$ will be considered in the next section.

\footnotetext{
${ }^{3}$ Ref. [61] computed $a_{R, u}$ for a closely-related model with coupling to $u_{R}$ rather than $d_{R}$ (the case referred to as mediator $M=\omega$ in that reference), but the result is the same in the present model

${ }^{4}$ One might have expected the opposite sign due to opposite weak isospins of $t_{L}$ and $\tilde{b}_{L}$, but this is reversed by the factor of $\not p p_{\mu} \rightarrow p^{2} \gamma_{\mu} / 4$ in the second diagram, which changes sign under Wick rotation.
} 
By $Z$ exchange, the operator (6) induces an anomalous contribution to the 4-quark effective operator

$$
\delta \mathcal{L}=\frac{G_{F}}{\sqrt{2}} \delta C_{1 d}\left(\bar{e} \gamma^{\mu} \gamma_{5} e\right)\left(\bar{d} \gamma_{\mu} d\right)
$$

where $\delta C_{1 d}=\left(a_{R, d}^{N P}+\delta a_{R, d}^{N P}\right)$, to be compared to its SM value $C_{1 d}=\frac{1}{2}-\frac{2}{3} \sin ^{2} \theta_{W}$. There is an analogous term with $C_{1 u}=-\frac{1}{2}+\frac{4}{3} \sin ^{2} \theta_{W}$ for the up quark, for which $\delta C_{1 u}=0$ in our model. The weak charge of a nucleus with $Z$ protons and $N$ neutrons is given by $Q_{W}(Z, N)=-2\left[(2 Z+N) C_{1 u}+(2 N+Z) C_{1 d}\right][64]$. A strong constraint on new physics contributions to APV comes from cesium $\left({ }^{133} \mathrm{Cs}\right)$, for which the weak charge is measured to be $Q_{W}(C s)=-73.20(35)$ [65-67], compared to the SM prediction of $Q_{W}^{S M}(C s)=$ $-73.15(2)$ [65]. Improved constraints are anticipated from the Qweak experiment [68] on the proton nuclear weak charge, measured via electron-proton scattering. Current data give $Q_{W}(p)=0.052(17)$, consistent with the expected value of $Q_{W}^{S M}(p)=0.0713(8)$ [69].

Further constraints on anomalous parity violation arise from neutrino deep inelastic scattering (DIS) experiments. In a similar manner to the nuclear weak charge case, the contribution of right-handed down quarks is described by the 4-fermion interaction

$$
\mathcal{L}_{\nu d}=\frac{G_{F}}{\sqrt{2}}\left(\epsilon_{R, d}+\delta \epsilon_{R, d}\right)\left(\bar{\nu} \gamma^{\mu}\left(1-\gamma_{5}\right) \nu\right)\left(\bar{d} \gamma_{\mu}\left(1+\gamma_{5}\right) d\right)
$$

where the SM contribution is $\epsilon_{R, d}=(2 / 3) \sin ^{2} \theta_{W}$ and the new physics contribution is $\delta \epsilon_{R, d}=a_{R, d}^{N P}+\delta a_{R, d}^{N P}$. Experiments in neutrino deep inelastic scattering provide measurements of the quantities $g_{R, L}^{2} \equiv \sum_{q} \epsilon_{R, L, q}^{2}$. Current data give $g_{R}^{2}=0.0309(10)$, in agreement with the SM prediction of $0.03001(2)$ [65]. These do not constrain our model as strongly as do the atomic cesium data.

\section{Numerical Analysis and Results}

In this section we describe our method of analysis and the resulting best-fit regions in the $R$ parity violating MSSM parameter space for fitting the FBA as well as top quark production data. Then we show how these regions are constrained by atomic parity violation.

\subsection{Analysis}

Following ref. [34], we distinguish the new physics (NP) contribution to the FBA from the SM contribution through the definition

$$
A_{F B}^{N P+S M}=\frac{\sigma_{F}^{N P}-\sigma_{B}^{N P}}{\sigma_{F}^{N P}+\sigma_{B}^{N P}+\sigma_{L O}^{S M}}+A_{F B}^{S M}\left(\frac{\sigma^{S M}}{\sigma^{S M}+\sigma^{N P}}\right),
$$

where the subscripts $F, B$ denote the forward and backward contributions to the cross section, respectively, and interference of the NP contribution with the LO SM term is 
taken into account in $\sigma_{F, B}^{N P}$, while the SM NLO+NNLO contributions are included in $\sigma^{S M}$ and $A_{F B}^{S M}$.

In addition to the inclusive asymmetry, we also consider the FBA coming from the low and high invariant mass regions. From ref. [7], the NLO + NNLL values for the SM contributions are $A_{F B}^{S M}=0.0724_{-0.00722}^{+0.0106}, A_{h}^{S M}=0.111_{-0.009}^{+0.017}$ and $A_{\ell}^{S M}=0.052_{-0.006}^{+0.009}$ respectively, and the NNLO value of the total SM cross-section is $\sigma^{S M}=\left(6.63_{-0.36}^{+0.33}\right) \mathrm{pb}$ [51]. The central values of the cross-section for the high and low invariant mass bins are taken to be $\sigma_{h}^{S M}=2.34 \mathrm{pb}$ and $\sigma_{l}^{S M}=4.29 \mathrm{pb}$. An overall $K$-factor of 1.4 was used to estimate higher-order QCD contributions to the cross-sections. $\sigma^{S M}$ includes the contribution of $g g$ fusion in addition to the dominant $q \bar{q}$ annihilation process. To maintain consistency with references [7] and [51], from which the SM predictions are taken, the MSTW2008NLO set of PDFs is used [52], evaluated at a scale $q=m_{t}=173.1 \mathrm{GeV}$. The PDF scale is varied between $m_{t} / 2$ and $2 m_{t}$ to give an approximate uncertainty on the calculated values. The top mass is taken to be $m_{t}=172.5 \mathrm{GeV}$ for the remainder of the analysis [70].

To compare the predictions to experimental values, the inclusive asymmetry is taken to be $A_{F B}^{t \bar{t}}=0.178 \pm 0.037$, obtained from a weighted average of the four values quoted in section 1 , adding the uncertainties in quadrature. The values of the folded, invariant mass dependent asymmetries are taken to be $A_{h}=0.296 \pm 0.067$ and $A_{\ell}=0.078 \pm 0.054$ [1]. The total production cross-section, $\sigma_{t \bar{t}}=(6.9 \pm 1.0) \mathrm{pb}$ and differential cross-section invariant mass distribution are as in ref. [50]; in particular, the value for the high-mass bin is $\left(d \sigma / d M_{t \bar{t}}\right)_{h} \equiv d \sigma / d M_{t \bar{t}}\left(800 \mathrm{GeV} \leq M_{t \bar{t}} \leq 1400 \mathrm{GeV}\right)=(0.068 \pm 0.032 \pm 0.015 \pm 0.004)$ $\mathrm{fb} \mathrm{GeV}^{-1}$.

We determine the allowed regions in the $\left(m_{\tilde{b}_{R}}, f_{d t}\right)$ parameter space (recall that squark mixing is neglected), by fitting the anomalous experimental values of the inclusive asymmetry and the corresponding low and high invariant mass bins, while maintaining agreement with the total and differential production cross-sections. Following [36] we construct a $\chi^{2}$ function in terms of these parameters, according to the naive definition

$$
\chi^{2}\left(m_{\tilde{b}}, f_{d t}\right)=\sum_{i} \frac{\left(\mathcal{O}_{i}\left(m_{\tilde{b}}, f_{d t}\right)-\mathcal{O}_{i}^{e x p}\right)^{2}}{\sigma_{i}^{2}}
$$

where $\mathcal{O}_{i}$ correspond to the Tevatron observables (we treat APV and LHC data separately below) and $\sigma_{i}$ their corresponding uncertainties, which are derived from the uncertainties on the experimental values and SM predictions, and those estimated from variation of the PDFs, by adding in quadrature. This gives a rough estimate of the allowed regions, since the true $\chi^{2}$ function should take into account the correlations between these observables through the covariance matrix; clearly the cross-section and the various asymmetries are correlated. However this is a much more involved task, and for this work we content ourselves with the estimate provided by (12).

An additional, separate $\chi^{2}$ is defined to fit LHC data, specifically the charge asymmetry in $t \bar{t}$ production and the relative differential top pair production cross-section, $1 / \sigma d \sigma / d M_{t \bar{t}}$, 
at high invariant masses. Although there is no forward-backward asymmetry at the LHC due to the fact that both beams are protons, the charge asymmetry is closely related, and is defined as

$$
A_{c}=\frac{\sigma(\Delta|y| \geq 0)-\sigma(\Delta|y| \leq 0)}{\sigma(\Delta|y| \geq 0)+\sigma(\Delta|y| \leq 0)}
$$

where $\Delta|y|=\left|y_{t}\right|-\left|y_{\bar{t}}\right|$. The NP and SM contributions to the charge asymmetry are distinguished as in (11). The measured value and SM prediction of the charge asymmetry are as given in sect. 1 .

We find that a much stronger constraint is posed by the differential cross-section, $1 / \sigma$ $d \sigma / d M_{t \bar{t}}$. We consider the relative differential cross-section corresponding to the two highest invariant mass bins, $700 \mathrm{GeV}<M_{t \bar{t}}<950 \mathrm{GeV}$ and $950 \mathrm{GeV}<M_{t \bar{t}}<2700 \mathrm{GeV}$, measured by ATLAS as $(0.24 \pm 0.04) \mathrm{TeV}^{-1}$ and $(0.007 \pm 0.003) \mathrm{TeV}^{-1}$, respectively [71]. Recently it was pointed out that electroweak Sudakov corrections lead to a reduction in the predicted cross section in models where the FBA is explained by new physics in the $t$-channel [72]. This reference showed that the reduction was approximately $10 \%$ at high invariant $t \bar{t}$ masses, in models where the new physics couples to left-handed quarks. It would be beyond the scope of the present work to recompute this correction for the case of interest, for new couplings to right-handed quarks. We simply assume it is the same and thus we apply a $10 \%$ downward correction to the predicted cross section.

We further define an analogous $\chi^{2}$ for the atomic parity violation data, using the three measurements of the Cs and proton weak charges and the neutrino DIS determination of

$g_{R}$, as discussed in sect. 3 . The resulting constraints on $\left(m_{\tilde{b}_{R}}, f_{d t}\right)$ are dominated by the Cs contribution.

\subsection{Results}

We find that the $R$-parity violating MSSM can generate a sufficient FBA, while maintaining agreement with the total production cross-section, to within $1 \sigma$. The dependence of both the asymmetry and production cross-section on $m_{\tilde{b}}$ is shown in Figure $3(\mathrm{a}, \mathrm{b})$, for various choices of the coupling $f_{d t}$. Conversely, the dependence of these quantities on the coupling is shown in Figure 3(c,d) for several values of $m_{\tilde{b}}$. There is a line of degeneracy in the $f_{d t}-m_{\tilde{b}}$ plane where a simultaneous fit can be achieved for both quantities, which can be seen in fig. 4. Here we plot the allowed intervals for the FBA and the production cross section together, showing that they overlap in a diagonal region starting near $m_{\tilde{b}}=400$ $\mathrm{GeV}, f_{d t}=2.4$, and continuing to higher masses and couplings along a band with slope $d f_{d t} / d m_{\tilde{b}} \cong 0.35 /(100 \mathrm{GeV})$. Fig. 4(a) shows the separate regions for the FBA and $\sigma_{t t}$ superimposed, while 4(b) shows the allowed regions for the low and high invariant mass parts of the FBA. Fig. 4(c) displays the overall preferred regions derived from the $\chi^{2}(12)$. The best-fit value is close to $m_{\tilde{b}}=600 \mathrm{GeV}, f_{d t}=3.2$, which we take as our benchmark model. 

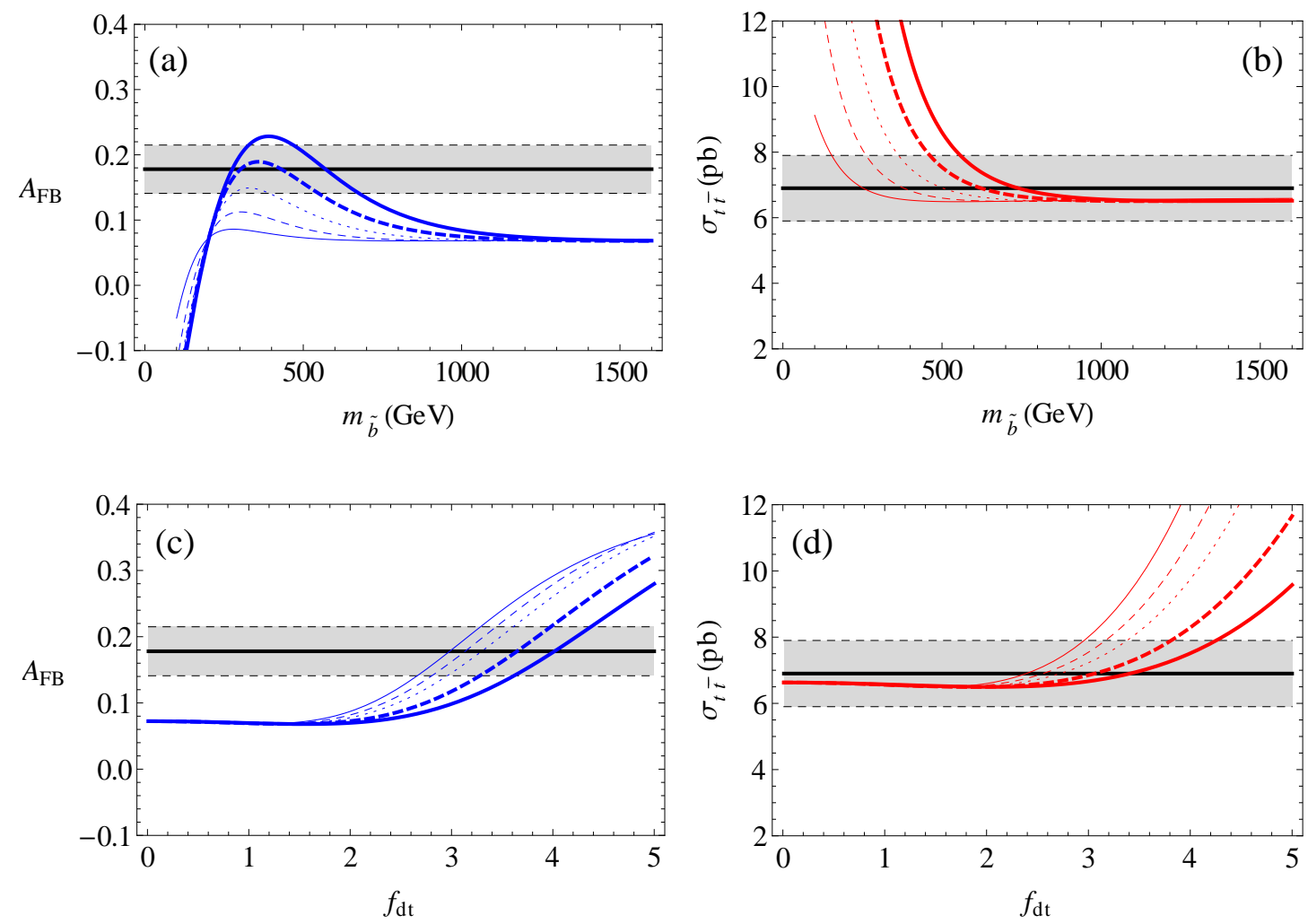

Figure 3: Figs. (a) and (b) show the sbottom mass dependence of the $t \bar{t}$ forward-backward asymmetry and $t \bar{t}$ production cross-section respectively, for various chosen values of the coupling $f_{d t}$. The solid, dashed, dotted, thick-dashed and thick-solid lines (i.e., going from bottom to top) correspond to $f_{d t}=\{1.6,2.0,2.4,2.8,3.2\}$. The experimental values are indicated by the black line, with the grey shaded regions corresponding to $1 \sigma$ deviation. Figs. (c) and (d) analogously show the dependence upon $f_{d t}$ for $m_{\tilde{b}}=\{500,550,600,700,800\}$ $\mathrm{GeV}$, going from top to bottom.

In addition to the inclusive asymmetry, we also find that the model gives a good simultaneous fit of the folded asymmetry values, corresponding to the high and low invariant mass bins, $M_{t \bar{t}}<450 \mathrm{GeV}$ and $M_{t \bar{t}} \geq 450 \mathrm{GeV}$. This is shown in fig. 5(a). The high and low mass asymmetries $A_{h}$ and $A_{\ell}$ are within $1 \sigma$ of the respective experimental values. The fit to the full set of collider observables is displayed in table 1 . The goodness of fit is determined, accounting for the total top pair production cross-section, the differential cross-section at high invariant masses $\left(800 \mathrm{GeV}<M_{t \bar{t}} \leq 1400 \mathrm{GeV}\right)$, the inclusive asymmetry, and the asymmetries at high and low invariant masses. The differential top pair production cross-section, with respect to the $t \bar{t}$ invariant mass, is presented in fig. $5(\mathrm{~b})$, 

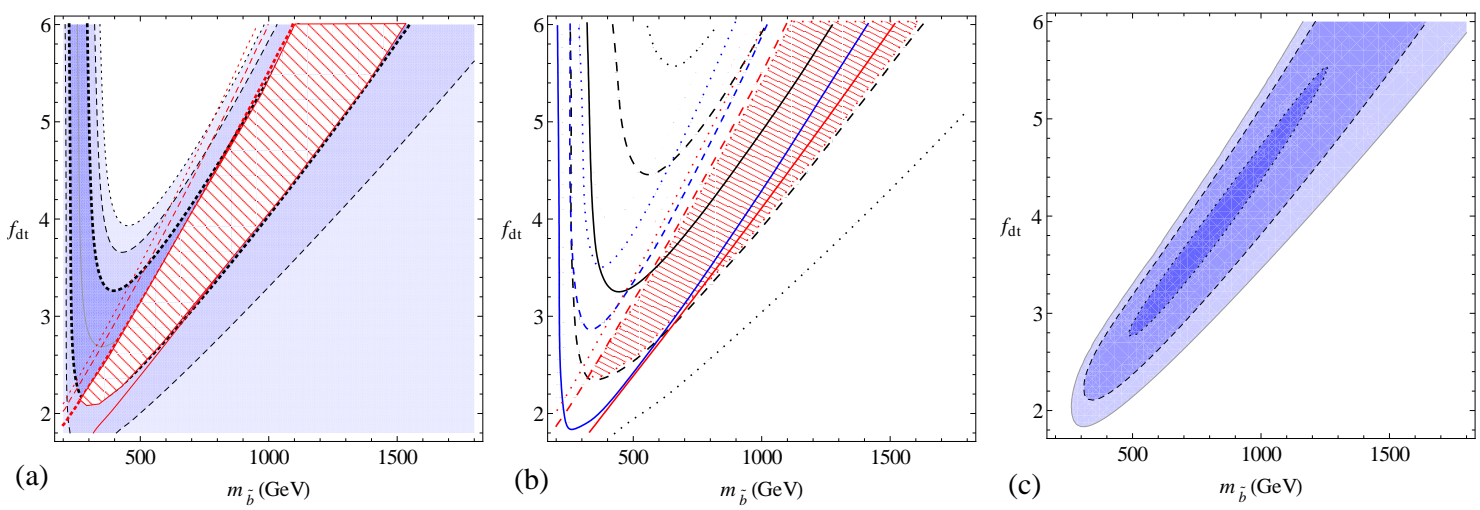

Figure 4: (a) Shaded regions are $68 \%, 95 \%$ and $99 \%$ confidence intervals in the $m_{\tilde{b}}-f_{d t}$ plane for FBA; (red) diagonal lines are central value and $68 \%, 95 \%, 99 \%$ c.l. upper limits on $f_{d t}$ for the production cross section; region of overlap of the $68 \%$ intervals is cross-hatched. (b) Same as (a), but for the low (blue, lower curves) and high (black, higher curves) invariant mass bins, and omitting the $99 \%$ contour. Solid lines indicate the central values. Overlap of all $68 \%$ intervals is cross-hatched. (c) Allowed 68\%, 95\% and 99\% c.l. regions from the combined $\chi^{2}$ for the FBA and the production cross section.

\begin{tabular}{|c|c|c|}
\hline & $\mathcal{O}_{i}^{e x p}$ & $\mathcal{O}_{i}\left(m_{\tilde{b}}=600 \mathrm{GeV}, f_{d t}=3.2\right)$ \\
\hline$A_{F B}^{t t}$ & $0.178 \pm 0.037$ & 0.167 \\
$A_{\ell}$ & $0.078 \pm 0.054$ & 0.107 \\
$A_{h}$ & $0.296 \pm 0.067$ & 0.252 \\
$\sigma_{t \bar{t}}$ & $(6.9 \pm 1.0) \mathrm{pb}$ & $7.54 \mathrm{pb}$ \\
$d \sigma_{t \bar{t}}^{h} / d M_{t \bar{t}}$ & $(0.068 \pm 0.032 \pm 0.015 \pm 0.004) \mathrm{fb} \mathrm{GeV}^{-1}$ & $0.097 \mathrm{fb} \mathrm{GeV}^{-1}$ \\
\hline
\end{tabular}

Table 1: Values of the fitted observables, for the benchmark model $\left(m_{\tilde{b}}=600 \mathrm{GeV}, f_{d t}=\right.$ 3.2) close to the minimum of the $\chi^{2}$.

while simultaneously fitting the full set of observables considered. It can be seen in the relevant plots that the results remain consistent with the experimental invariant mass distribution. Moreover, the problem of the enhancement of the tail of the distribution, which previously plagued $t$-channel models of this kind $[29,34,53,54]$ is resolved by using the updated value of $A_{h}$ [1]. Both of these figures and the table refer to the benchmark model $\left(m_{\tilde{b}}=600 \mathrm{GeV}, f_{d t}=3.2\right)$.

We find that there is some tension between this model and LHC data, namely the relative differential cross-section, as enhancement of the cross-section at high invariant masses disfavors a significant region of parameter space. In fig. 7 we show the region of parameter space allowed by the LHC-measured observables. Although the 1- $\sigma$ allowed regions from the two experiments do not intersect, there is considerable overlap between 

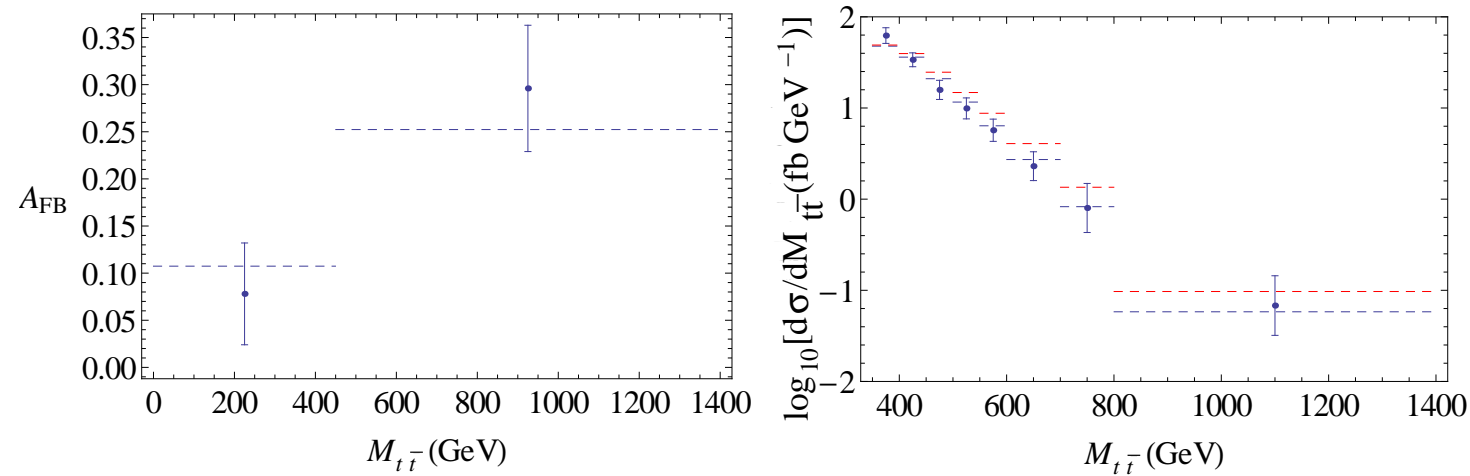

Figure 5: (a) Left: the invariant mass-dependent asymmetry; the values of the high and low mass bin are displayed both for the CDF result, shown with errors, and the calculated value, represented by the dashed line. Benchmark values of $m_{\tilde{b}}=600 \mathrm{GeV}, f_{d t}=3.2$ were chosen, corresponding to the minimum of the total $\chi^{2}$. (b) Right: binned invariant mass distribution of the differential $t \bar{t}$ production cross section, displaying both the CDF results (shown with error bars) and the calculated result, represented by the dashed lines. The lower (blue) dashed line shows the result calculated assuming only the standard model process while the upper (red) includes the new physics contribution from squark exchange. The same model parameters are assumed as in (a).

the 2- $\sigma$ allowed regions, spanning sbottom masses from 300 to $900 \mathrm{GeV}$ and couplings $f_{d t}$ from 2.4 to 3.6. Thus although the overall fit from combining Tevatron and LHC is not ideal, the model is far from being ruled out. We differ in this conclusion from ref. [73], which considered more generally the constraints on models with color-triplet exchange. In that work, an effective operator approach was used, where the heavy mediator particle was integrated out, which may not be justified for the low squark masses considered here. Other differences between the two analyses is our use of more recent ATLAS data [71] and taking into account electroweak Sudakov corrections [72]. On the other hand, the constraint from the charge asymmetry is relatively weak, and we find the model to be consistent to within $1 \sigma$ with this measurement over the entire parameter space considered. As the most recent LHC determinations of the charge asymmetry are dominated by both statistical and systematic uncertainty, it is not yet at the level where it can provide significant constraints on models that predict an enhanced FBA.

In ref. [61], it was shown that the absence of anomalous parity violation in Cs rules out the explanation of the top quark FBA in some models that are closely related to the present one. We confirm that this is also the case for the $R$-parity violating MSSM, if only the leftmost diagram of fig. 2 contributes significantly to the $\bar{d}_{R} \psi_{d_{R}}$ coupling. Fig. 6(a) shows that the upper limits (diagonal lines) on the coupling $f_{d t}$ as a function of $m_{\tilde{b}}$ are well below the values required to explain the FBA in this case. However as we noted in 

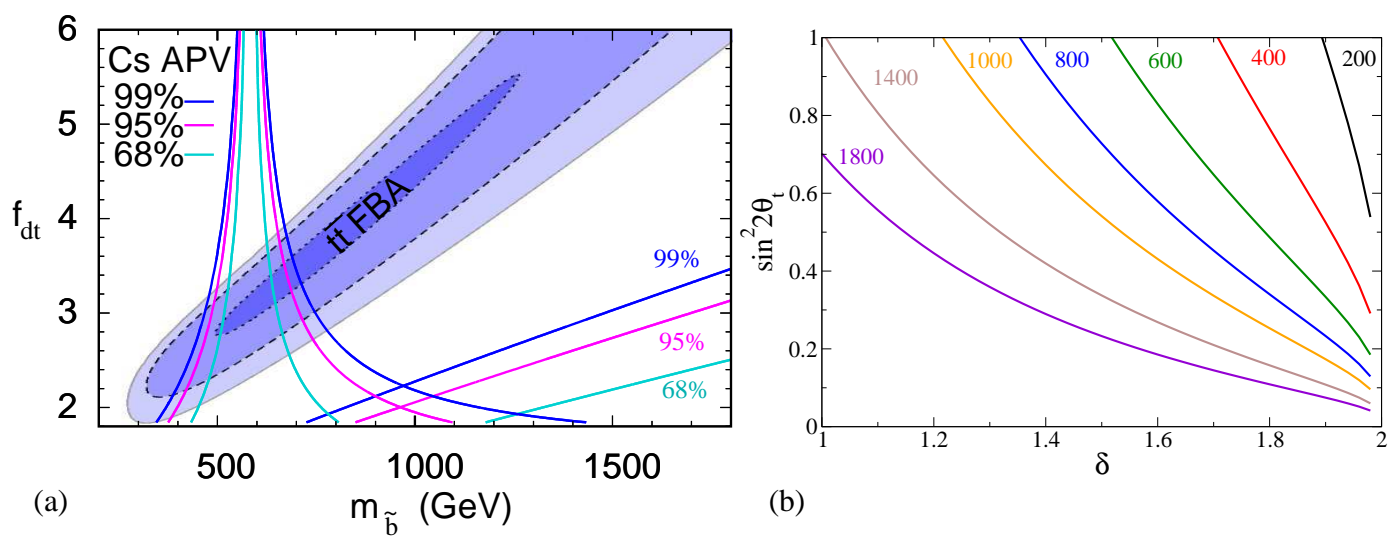

(b)

Figure 6: (a) Left: upper limits on $f_{d t}$ from Cesium atomic parity violation at $68 \%, 95 \%$ and $99 \%$ c.l., superimposed on the (shaded) regions allowed by the top quark FBA and production cross section. The lower set of curves (straight lines) assumes a generic squark spectrum, while the upper set requires large top squark mixing $\sin ^{2} 2 \theta=0.5$ and mass splitting $\delta \equiv\left(m_{\tilde{t}_{1}}^{2}-m_{\tilde{t}_{2}}^{2}\right) /\left[\frac{1}{2}\left(m_{\tilde{t}_{1}}^{2}+m_{\tilde{t}_{2}}^{2}\right)\right]=1.8$. (b) Right: relation between $\sin ^{2} 2 \theta_{\tilde{t}}$ and $\delta$ needed for nullification of the Cs constraint, for different values of $m_{\tilde{b}}$ as labeled on the figure, in $\mathrm{GeV}$.

section 3, the extra diagram with $Z$ coupling to $\tilde{t}$ is generically present and contributes with the opposite sign. To get a large cancellation with the first diagram requires both large mixing and large mass splitting of the top squarks. In Fig. 6(a) we show an example of the weakened constraints obtained by taking $\sin ^{2} 2 \theta_{\tilde{t}}=0.5$ and stop masses split by $\delta=1.8$, corresponding to $m_{\tilde{t}_{1,2}}=(0.32,1.38) m_{0}$. (The overall scale $m_{0} \gg m_{b}$ is irrelevant for the value of the rightmost diagram in fig. 2, which is dimensionless.) We see in this example that the cancellation is complete for $m_{\tilde{b}} \cong 600 \mathrm{GeV}$ and fully allows for our benchmark model. More generally, the value of $m_{\tilde{b}}$ for which the cancellation is maximized depends upon the stop mixing and mass splitting, and moves to higher values as we take smaller splitting. It is determined by $F_{1}\left(m_{t}^{2} / m_{\tilde{b}}^{2}\right)=2 \sin ^{2} 2 \theta_{\tilde{t}} F_{2}(\delta)$. This relation is plotted in the plane of $\sin ^{2} 2 \theta_{\tilde{t}}$ and $\delta$ for the relevant range bottom squark masses in fig. 6(b). Thus one can achieve consistency with the APV constraints over a range of reasonable values of the parameters.

\section{Conclusion}

The anomalously large top pair forward-backward asymmetry, observed by both CDF and $\mathrm{D} \emptyset$ is suggestive of new physics, although new models are subject to numerous constraints from collider data, namely the strong agreement between the SM prediction and experimental result in the total $t \bar{t}$ production cross-section. The process $d \bar{d} \rightarrow t \bar{t}$, via sbottom 


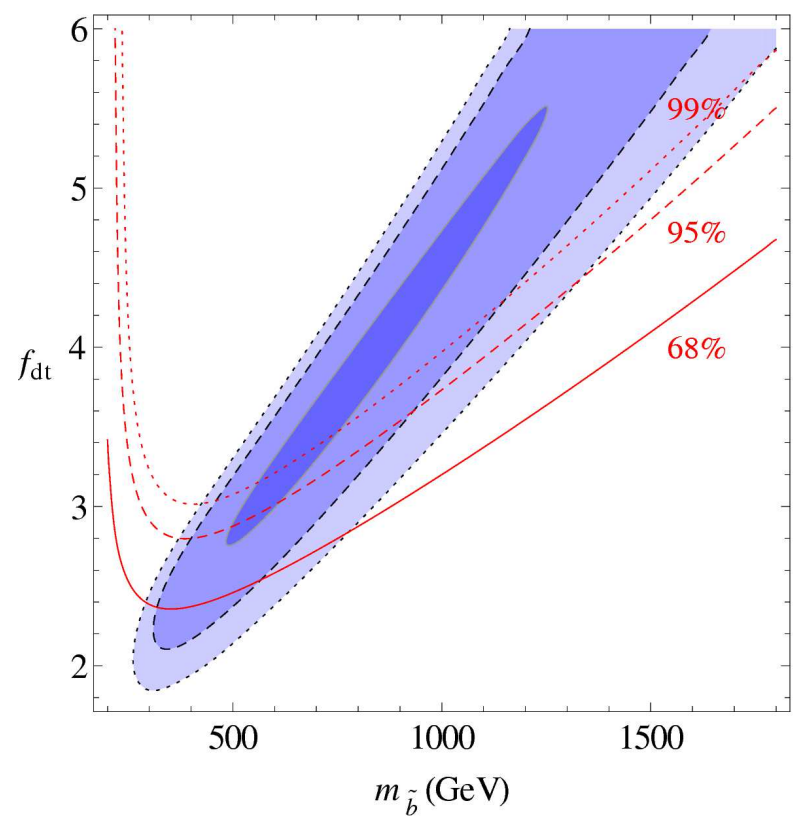

Figure 7: (a) Upper limits on the relative differential cross section for $t \bar{t}$ production from LHC data at $68 \%, 95 \%$ and $99 \%$ c.l., superimposed on the (shaded) regions allowed by the Tevatron top quark FBA and production cross-section.

exchange in $R$-parity violating supersymmetry with the interaction $\tilde{b}_{R}^{*} \bar{t}_{R} d_{R}^{c}$, has been proposed as a mechanism to account for the FBA. We find that the model can produce the required asymmetry, while preserving agreement with the total production cross-section. Moreover, results remain consistent with the differential cross-section, even at high invariant masses. 
However, there is some tension between the model and LHC data. Although the model preserves agreement with the measured value of the charge asymmetry, a large region of the parameter space allowed by simultaneously fitting the Tevatron observables is disfavored due to enhancement of the relative differential cross-section at high invariant masses. Nevertheless there is overlap between the $2-\sigma$ allowed regions of the FBA and the ATLAS top production cross section, leaving $R$-parity violating SUSY as possible explanation of the anomaly. The agreement is improved somewhat by taking into account electroweak Sudakov corrections [72], which we have done in an approximate way. A more exact computation of this effect for new physics coupling to right-handed quarks, as appropriate to the present model, could be interesting for future study.

The constraints posed by atomic parity violation in Cesium would naively appear to exclude this explanation, but we have shown that a canceling contribution to the offending one is naturally present due to the operator $f_{d t} \tilde{t}_{R}^{*} \bar{b}_{R} d_{R}^{c}$ that automatically comes with the same strength as $f_{d t} \tilde{b}_{R}^{*} \bar{t}_{R} d_{R}^{c}$ in the R-parity violating MSSM, and it can nullify the the effect of the latter for large but reasonable values of the stop mixing and mass splitting. This loophole can also be compatible with a constraint on $f_{d t}$ from neutron-antineutron oscillations [59] if the sbottom quark mixing is small or the Wino mass is large. It could be interesting to quantify this statement in light of the allowed region of parameter space we identify in this work.

An interesting feature of the model could be its implication to $b$ physics, since the extra operator $f_{d t} \tilde{t}_{R}^{*} \bar{b}_{R} d_{R}^{c}$ implies analogous new contributions to $b \bar{b}$ production, leading to further constraints and possible predictions for future experimental results. One expects to observe a forward-backward asymmetry in $b \bar{b}$ production at a comparable level to that of $t \bar{t}$.

A possible concern with this model is the existence of large flavor-changing neutral currents. ${ }^{5}$ For example, there is a box diagram for $B_{d}-\bar{B}_{d}$ mixing with $t, \tilde{b}$ and $\tilde{d}$ in the loop which induces a contribution to the effective operator $\Lambda^{-2}\left(\bar{b}_{R} \gamma_{\mu} d_{R}\right)\left(\bar{d}_{R} \gamma_{\mu} b_{R}\right)$ with $\Lambda \cong 8 \pi m_{\tilde{q}} / f_{d t}^{2}$ assuming a common squark mass $m_{\tilde{q}}$. For our benchmark model, this would give $\Lambda=1.5 \mathrm{TeV}$, which is far below the limit of $200 \mathrm{TeV}$ for models with no GIM suppression (though close to the limit of $1.8 \mathrm{TeV}$ for models having minimal or next-to-minimal flavour violating structure) [74]. However, this outcome can be avoided if $m_{\tilde{d}} \ggg m_{\tilde{b}}$. There are well-motivated SUSY scenarios in which only the third generation is light [75-78], and it has been argued that $R$-parity violation is particularly natural within this framework $[79,80]$. A complete study of FCNC constraints on our model in this case is beyond the scope of this paper, but would be interesting to undertake.

After completion of this work, we became aware of ref. [81], which also includes a study of the FBA in the $R$-parity violating MSSM. There the constraint $\left|f_{d t}\right|<1.25$ was imposed, leading to a negative conclusion. This was based upon requiring $f_{d t}$ to remain perturbative up to the unification scale [82]. With couplings $f_{d t} \gtrsim 2.8$ as we favor for the

\footnotetext{
${ }^{5}$ we realized this problem as we were finishing the present paper
} 
FBA, such a requirement must be abandoned in favor of the more modest one that $f_{d t}$ does not reach a Landau pole at too low a scale. The paradigm of very heavy first and second generation squarks may help in this respect, since $f_{d t}$ runs more slowly in their absence, with $d f_{d t} / d t \cong 3 f_{d t}^{2} / 16 \pi^{2}$, ignoring gauge couplings. We estimate the Landau pole scale to be $\Lambda \sim 14 \mathrm{TeV}$ for the case $f_{d t}=2.8, m_{\tilde{b}}=500 \mathrm{GeV}$, going up to $\Lambda \sim 70 \mathrm{TeV}$ for $f_{d t}=2.2, m_{\tilde{b}}=300 \mathrm{GeV}$ (the lower boundary of the $95 \%$ c.l. region).

\section{Acknowledgements}

We thank Mike Trott for valuable guidance and suggestions at the beginning of this project. We also thank Sean Tulin for very helpful correspondence. JC thanks the Aspen Center for Physics where discusssions pertinent to this work took place. GD is supported by Tomlinson and McGill Majors Fellowships. JC is supported by Natural Sciences and Engineering Research Council, Canada.

\section{A Computation of FBA}

The matrix element for the process $d \bar{d} \rightarrow t \bar{t}$, including the SM contribution by $s$-channel gluon exchange, and neglecting the $\tilde{b}$ width (which we reinstate below) is given by

$$
\begin{aligned}
\mathcal{M}_{S M+\tilde{b}}= & \frac{g_{s}^{2}}{\hat{s}}\left[\bar{v}\left(p_{\bar{d}}\right) \gamma_{\mu} t^{a} u\left(p_{d}\right)\right]\left[\bar{u}\left(p_{t}\right) \gamma^{\mu} t^{a} v\left(p_{\bar{t}}\right)\right] \\
& -\frac{\left|f_{d t}\right|^{2}}{2\left(\hat{u}-m_{\tilde{b}}^{2}\right)}\left\{\left[\bar{v}\left(p_{\bar{d}}\right) \gamma_{\mu} P_{R} u\left(p_{d}\right)\right]\left[\bar{u}\left(p_{t}\right) \gamma^{\mu} P_{R} v\left(p_{\bar{t}}\right)\right]\right. \\
& \left.-\left[\bar{v}_{\alpha}\left(p_{\bar{d}}\right) \gamma_{\mu} P_{R} u^{\beta}\left(p_{d}\right)\right]\left[\bar{u}_{\beta}\left(p_{t}\right) \gamma^{\mu} P_{R} v^{\alpha}\left(p_{\bar{t}}\right)\right]\right\}
\end{aligned}
$$

where $\hat{s}$ and $\hat{u}$ are the Mandelstam variables in the partonic centre of mass frame and the quark momenta are taken as

$$
\begin{aligned}
& p_{d, \bar{d}}=\frac{\sqrt{\hat{s}}}{2}(1,0,0, \pm 1) \\
& p_{t, \bar{t}}=\frac{\sqrt{\hat{s}}}{2}(1, \pm \beta \sin \theta, 0, \pm \beta \cos \theta),
\end{aligned}
$$


with $\beta^{2}=1-4 m_{t}^{2} / \hat{s}$ and where $\theta$ is the scattering angle in the partonic centre of mass frame. Squaring and summing over colour and spin gives

$$
\begin{aligned}
\left|\mathcal{M}_{S M+\tilde{b}}\right|^{2}= & 4 \pi^{2} \alpha_{s}^{2}\left(\frac{N_{c}^{2}-1}{N_{c}^{2}}\right)\left(1+\beta^{2} \cos ^{2} \theta+\frac{4 m_{t}^{2}}{\hat{s}}\right) \\
& -\frac{\pi \alpha_{s}}{2}\left(\frac{N_{c}^{2}-1}{N_{c}^{2}}\right)\left(\frac{\left|f_{d t}\right|^{2} \hat{s}}{\hat{u}-m_{\tilde{b}}^{2}}\right)\left((1+\beta \cos \theta)^{2}+\frac{4 m_{t}^{2}}{\hat{s}}\right) \\
& +\frac{1}{8}\left(\frac{N_{c}-1}{N_{c}}\right)\left(\frac{\left|f_{d t}\right|^{2} \hat{s}}{\hat{u}-m_{\tilde{b}}^{2}}\right)^{2}(1+\beta \cos \theta)^{2},
\end{aligned}
$$

with $N_{c}=3$. Accounting for resonance near the sbottom mass, the matrix element becomes

$$
\begin{aligned}
\left|\mathcal{M}_{S M+\tilde{b}}\right|^{2}= & \frac{32 \pi^{2} \alpha_{s}^{2}}{9}\left(1+\beta^{2} \cos ^{2} \theta+\frac{4 m_{t}^{2}}{\hat{s}}\right) \\
& -\frac{4 \pi \alpha_{s}\left|f_{d t}\right|^{2}}{9} \frac{\hat{s}\left(\hat{u}-m_{\tilde{b}}^{2}\right)\left(1+\beta^{2} \cos ^{2} \theta+\frac{4 m_{t}^{2}}{\hat{s}}\right)}{\left(\hat{u}-m_{\tilde{b}}^{2}\right)^{2}+\Gamma^{2} m_{\tilde{b}}^{2}} \\
& +\frac{\left|f_{d t}\right|^{4} \hat{s}^{2}}{12} \frac{(1+\beta \cos \theta)^{2}}{\left(\hat{u}-m_{\tilde{b}}^{2}\right)^{2}+\Gamma^{2} m_{\tilde{b}}^{2}} .
\end{aligned}
$$

The sbottom decay width in this model is dominated by $\tilde{b} \rightarrow \bar{t} \bar{d}$, giving $\Gamma \cong\left|f_{d t}\right|^{2}\left(m_{\tilde{b}} / 8 \pi\right)$ $\times\left(1-m_{t}^{2} / m_{\tilde{b}}^{2}\right)^{2}$. The differential cross-section is then given by

$$
\frac{d \hat{\sigma}}{d \cos \theta}=\frac{\beta}{32 \pi s}\left|\mathcal{M}_{S M+\tilde{b}}\right|^{2}
$$

with the hat denoting the partonic quantity in the usual convention. The forward-backward asymmetry, defined as

$$
A_{F B}=\frac{\sigma(\Delta y \geq 0)-\sigma(\Delta y \leq 0)}{\sigma(\Delta y \geq 0)+\sigma(\Delta y \leq 0)}
$$

where $\Delta y$ is the rapidity of the top quarks, $y_{t}-y_{\bar{t}}$, is then given in the $t \bar{t}$ centre of mass frame by

$$
A_{F B}^{t \bar{t}}=\frac{\sigma_{F}-\sigma_{B}}{\sigma_{F}+\sigma_{B}}
$$

where $\sigma_{F}$ and $\sigma_{B}$ are the forward and backward hadronic cross-sections, evaluated in the $t \bar{t}$ frame, and given by

$$
\sigma_{F}=\int_{0}^{1} d \cos \theta \frac{d \sigma}{d \cos \theta}, \quad \sigma_{B}=\int_{-1}^{0} d \cos \theta \frac{d \sigma}{d \cos \theta}
$$


with $\Delta y=2 \tanh ^{-1}(\beta \cos \theta)$. The hadronic cross-sections are obtained by convolutions with the PDFs, according to

$$
\frac{d \sigma}{d M_{t \bar{t}}}=\frac{M_{t \bar{t}}}{s} \int_{-y_{B}^{0}}^{y_{B}^{0}} d y_{B} \int_{-1}^{1} d z f_{d}\left(\tau e^{y_{B}}\right) f_{d}\left(\tau e^{-y_{B}}\right) \frac{d \hat{\sigma}}{d z}
$$

where $s$ is the hadronic centre of mass energy, $y_{B}$ is the boost rapidity of the subprocess

frame, $\tau=\sqrt{\frac{M_{t \bar{t}}^{2}}{s}}, z=\cos \theta$ and $f_{i}$ are the parton distribution functions. In accordance with [83], an experimental rapidity cut $\left|y_{1,2}\right|<y_{\max }$ corresponds to the integration limits

$$
y_{B}^{0}=\min \left[y_{\max },-\ln \tau\right] .
$$

\section{References}

[1] S. Leone, "Top Quark Production at the Tevatron," in Rencontres de Moriond, EW. 2012.

http://indico.in2p3.fr/getFile.py/access?contribId=22\&sessionId=9\&resId=0\&materialId=sl

[2] CDF Collaboration Collaboration, T. Aaltonen et al., "Evidence for a Mass Dependent Forward-Backward Asymmetry in Top Quark Pair Production," Phys.Rev. D83 (2011) 112003, arXiv:1101.0034 [hep-ex]. 23 pages, 18 figures, submitted to Physical Review D.

[3] CDF Collaboration Collaboration, T. Aaltonen et al., "Forward-Backward Asymmetry in Top Quark Production in $p \bar{p}$ Collisions at $\sqrt{s}=1.96 \mathrm{TeV}$," Phys.Rev.Lett. 101 (2008) 202001, arXiv:0806.2472 [hep-ex].

[4] CDF Collaboration, Y. Takeuchi et al., "Measurement of the Forward Backward Asymmetry in Top Pair Production in the Dilepton Decay Channel using $5.1 \mathrm{fb}^{-1}$," 2007. http://www-cdf.fnal.gov/physics/new/top/2011/DilAfb/.

[5] Do Collaboration Collaboration, V. M. Abazov et al., "Forward-backward asymmetry in top quark-antiquark production," Phys.Rev. D84 (2011) 112005, arXiv:1107.4995 [hep-ex].

[6] Do Collaboration Collaboration, R. Demina, "Measurement of the forward-backward charge asymmetry in top quark production in proton-antiproton collisions at $1.96 \mathrm{TeV}, "$ in EPS-HEP Conference, Grenoble. 2011. http://pos.sissa.it/archive/conferences/134/379/EPS-HEP2011_379.pdf.

[7] V. Ahrens, A. Ferroglia, M. Neubert, B. D. Pecjak, and L. L. Yang, "The top-pair forward-backward asymmetry beyond NLO," Phys.Rev. D84 (2011) 074004, arXiv:1106.6051 [hep-ph]. 
[8] O. Antunano, J. H. Kuhn, and G. Rodrigo, "Top quarks, axigluons and charge asymmetries at hadron colliders," Phys.Rev. D77 (2008) 014003, arXiv:0709.1652 [hep-ph].

[9] L. Sehgal and M. Wanninger, "Forward-Backward Asymmetry in Two Jet Events: Signature of Axigluons in Proton-Antiproton Collisions,"

Phys.Lett. B200 (1988) 211.

[10] J. Bagger, C. Schmidt, and S. King, "AxigluonbProduction in Hadronic Collisions," Phys.Rev. D37 (1988) 1188.

[11] P. Ferrario and G. Rodrigo, "Constraining heavy colored resonances from top-antitop quark events," Phys.Rev. D80 (2009) 051701, arXiv:0906.5541 [hep-ph].

[12] P. H. Frampton, J. Shu, and K. Wang, "Axigluon as Possible Explanation for $p \bar{p} \rightarrow t \bar{t}$ Forward-Backward Asymmetry," Phys.Lett. B683 (2010) 294-297, arXiv:0911.2955 [hep-ph]. revtex. 5 pages, 2 figures.

[13] R. S. Chivukula, E. H. Simmons, and C.-P. Yuan, "Axigluons cannot explain the observed top quark forward-backward asymmetry," Phys.Rev. D82 (2010) 094009, arXiv:1007.0260 [hep-ph].

[14] Y. Bai, J. L. Hewett, J. Kaplan, and T. G. Rizzo, "LHC Predictions from a Tevatron Anomaly in the Top Quark Forward-Backward Asymmetry," JHEP 1103 (2011) 003, arXiv:1101.5203 [hep-ph].

[15] A. R. Zerwekh, "The Axigluon, a Four-Site Model and the Top Quark Forward-Backward Asymmetry at the Tevatron," Phys.Lett. B704 (2011) 62-65, arXiv:1103.0956 [hep-ph].

[16] U. Haisch and S. Westhoff, "Massive Color-Octet Bosons: Bounds on Effects in Top-Quark Pair Production," JHEP 1108 (2011) 088, arXiv:1106.0529 [hep-ph].

[17] G. M. Tavares and M. Schmaltz, "Explaining the $t \bar{t}$ asymmetry with a light axigluon," Phys.Rev. D84 (2011) 054008, arXiv:1107.0978 [hep-ph].

[18] E. Alvarez, L. Da Rold, J. I. S. Vietto, and A. Szynkman, "Phenomenology of a light gluon resonance in top-physics at Tevatron and LHC," JHEP 1109 (2011) 007, arXiv:1107.1473 [hep-ph].

[19] J. Aguilar-Saavedra and M. Perez-Victoria, "Shaping the top asymmetry," Phys.Lett. B705 (2011) 228-234, arXiv:1107.2120 [hep-ph].

[20] A. Djouadi, G. Moreau, F. Richard, and R. K. Singh, "The Forward-backward asymmetry of top quark production at the Tevatron in warped extra dimensional models," Phys.Rev. D82 (2010) 071702, arXiv:0906.0604 [hep-ph]. 
[21] M. Bauer, F. Goertz, U. Haisch, T. Pfoh, and S. Westhoff, "Top-Quark Forward-Backward Asymmetry in Randall-Sundrum Models Beyond the Leading Order," JHEP 1011 (2010) 039, arXiv:1008.0742 [hep-ph].

[22] C. Delaunay, O. Gedalia, S. J. Lee, G. Perez, and E. Ponton, "Extraordinary Phenomenology from Warped Flavor Triviality," Phys.Lett. B703 (2011) 486-490, arXiv:1101.2902 [hep-ph].

[23] S. C. Park and J. Shu, "Split Universal Extra Dimensions and Dark Matter," Phys.Rev. D79 (2009) 091702, arXiv:0901.0720 [hep-ph].

[24] C.-H. Chen, G. Cvetic, and C. Kim, "Forward-backward asymmetry of top quark in unparticle physics," Phys.Lett. B694 (2011) 393-397, arXiv:1009.4165 [hep-ph].

[25] E. Alvarez, L. Da Rold, and A. Szynkman, "A composite Higgs model analysis of forward-backward asymmetries in the production of tops at Tevatron and bottoms at LEP and SLC," JHEP 1105 (2011) 070, arXiv:1011.6557 [hep-ph].

[26] E. R. Barreto, Y. Coutinho, and J. Sa Borges, "Top quark forward-backward asymmetry from the 3-3-1 model," Phys.Rev. D83 (2011) 054006, arXiv:1103.1266 [hep-ph].

[27] R. Foot, "Top quark forward-backward asymmetry from $\mathrm{SU}\left(N_{c}\right)$ color," Phys.Rev. D83 (2011) 114013, arXiv:1103.1940 [hep-ph].

[28] J. F. Kamenik, J. Shu, and J. Zupan, "Review of new physics effects in $t \bar{t}$ production," arXiv:1107.5257 [hep-ph].

[29] Z. Ligeti, G. M. Tavares, and M. Schmaltz, "Explaining the $t \bar{t}$ forward-backward asymmetry without dijet or flavor anomalies," JHEP 1106 (2011) 109, arXiv:1103.2757 [hep-ph].

[30] B. Grinstein, A. L. Kagan, M. Trott, and J. Zupan, "Forward-backward asymmetry in $t \bar{t}$ production from flavour symmetries," Phys.Rev.Lett. 107 (2011) 012002, arXiv:1102.3374 [hep-ph].

[31] A. E. Nelson, T. Okui, and T. S. Roy, "A unified, flavor symmetric explanation for the $t \bar{t}$ asymmetry and Wjj excess at CDF," Phys.Rev. D84 (2011) 094007, arXiv:1104.2030 [hep-ph]. minor changes to text, references added, version to be published in PRD.

[32] C. W. Bauer, Z. Ligeti, M. Schmaltz, J. Thaler, and D. G. Walker, "Supermodels for early LHC," Phys.Lett. B690 (2010) 280-288, arXiv:0909.5213 [hep-ph].

[33] J. M. Arnold, M. Pospelov, M. Trott, and M. B. Wise, "Scalar Representations and Minimal Flavor Violation," JHEP 1001 (2010) 073, arXiv:0911.2225 [hep-ph]. 
[34] B. Grinstein, A. L. Kagan, J. Zupan, and M. Trott, "Flavor Symmetric Sectors and Collider Physics," JHEP 1110 (2011) 072, arXiv:1108.4027 [hep-ph].

[35] N. Craig, C. Kilic, and M. J. Strassler, "LHC Charge Asymmetry as Constraint on Models for the Tevatron Top Anomaly," Phys.Rev. D84 (2011) 035012, arXiv:1103.2127 [hep-ph].

[36] B. Bhattacherjee, S. S. Biswal, and D. Ghosh, "Top quark forward-backward asymmetry at Tevatron and its implications at the LHC,"

Phys.Rev. D83 (2011) 091501, arXiv:1102.0545 [hep-ph].

[37] S. Jung, H. Murayama, A. Pierce, and J. D. Wells, "Top quark forward-backward asymmetry from new t-channel physics," Phys.Rev. D81 (2010) 015004, arXiv:0907.4112 [hep-ph].

[38] P. Ko, Y. Omura, and C. Yu, "Top forward-backward asymmetry and the CDF Wjj excess in leptophobic U(1)' flavor models," arXiv:1108.0350 [hep-ph].

[39] K. Cheung, W.-Y. Keung, and T.-C. Yuan, "Top Quark Forward-Backward Asymmetry," Phys.Lett. B682 (2009) 287-290, arXiv:0908.2589 [hep-ph].

[40] B. Xiao, Y. kai Wang, and S. hua Zhu, "Forward-backward Asymmetry and Differential Cross Section of Top Quark in Flavor Violating Z' model at $\mathcal{O}\left(\alpha_{s}^{2} \alpha_{X}\right)$," Phys.Rev. D82 (2010) 034026, arXiv:1006.2510 [hep-ph].

[41] K. Cheung and T.-C. Yuan, "Top Quark Forward-Backward Asymmetry in the Large Invariant Mass Region," Phys.Rev. D83 (2011) 074006, arXiv:1101.1445 [hep-ph].

[42] E. L. Berger, Q.-H. Cao, C.-R. Chen, C. S. Li, and H. Zhang, "Top Quark Forward-Backward Asymmetry and Same-Sign Top Quark Pairs," Phys.Rev.Lett. 106 (2011) 201801, arXiv:1101.5625 [hep-ph].

[43] M. Duraisamy, A. Rashed, and A. Datta, "The Top Forward Backward Asymmetry with general Z' couplings," Phys.Rev. D84 (2011) 054018, arXiv:1106.5982 [hep-ph]. 13 pages, 14 figures, minor typos corrected, accepted for publication in Physical Review D.

[44] J. Cao, L. Wang, L. Wu, and J. M. Yang, "Top quark forward-backward asymmetry, FCNC decays and like-sign pair production as a joint probe of new physics," Phys.Rev. D84 (2011) 074001, arXiv:1101.4456 [hep-ph].

[45] C.-H. Chen, S. S. Law, and R.-H. Li, "Rare B decays and Tevatron top-pair asymmetry," J.Phys.G G38 (2011) 115008, arXiv:1104.1497 [hep-ph]. 
[46] V. Barger, W.-Y. Keung, and C.-T. Yu, "Tevatron Asymmetry of Tops in a W', Z' Model," Phys.Lett. B698 (2011) 243-250, arXiv:1102.0279 [hep-ph].

[47] M. Frank, A. Hayreter, and I. Turan, "Top Quark Pair Production and Asymmetry at the Tevatron and LHC in Left-Right Models," Phys.Rev. D84 (2011) 114007, arXiv:1108.0998 [hep-ph].

[48] V. Barger, W.-Y. Keung, and C.-T. Yu, "Asymmetric Left-Right Model and the Top Pair Forward-Backward Asymmetry," Phys.Rev. D81 (2010) 113009, arXiv:1002.1048 [hep-ph].

[49] J. Shelton and K. M. Zurek, "Maximal flavor violation from new right-handed gauge bosons," Phys.Rev. D83 (2011) 091701, arXiv:1101.5392 [hep-ph].

[50] CDF Collaboration Collaboration, T. Aaltonen et al., "First Measurement of the $t \bar{t}$ Differential Cross Section $\frac{d \sigma}{d M_{t \bar{t}}}$ in $p \bar{t}$ Collisions at $\sqrt{s}=1.96 \mathrm{TeV}, "$ Phys.Rev.Lett. 102 (2009) 222003, arXiv:0903.2850 [hep-ex].

[51] V. Ahrens, A. Ferroglia, M. Neubert, B. D. Pecjak, and L.-L. Yang, "RG-improved single-particle inclusive cross sections and forward-backward asymmetry in $t \bar{t}$ production at hadron colliders," JHEP 1109 (2011) 070, arXiv:1103.0550 [hep-ph].

[52] A. Martin, W. Stirling, R. Thorne, and G. Watt, "Parton distributions for the LHC," Eur.Phys.J. C63 (2009) 189-285, arXiv:0901.0002 [hep-ph].

[53] M. I. Gresham, I.-W. Kim, and K. M. Zurek, "On Models of New Physics for the Tevatron Top $A_{F B}$," Phys.Rev. D83 (2011) 114027, arXiv:1103.3501 [hep-ph].

[54] K. Blum, Y. Hochberg, and Y. Nir, "Scalar-mediated $t \bar{t}$ forward-backward asymmetry," JHEP 1110 (2011) 124, arXiv:1107.4350 [hep-ph]. 22 pages, 1 figure and 2 tables. v2: Corrected Eqs.(50,51,74), adapted Fig.1, Tab.1 and relevant discussions. Extended discussion of top decay and single top.

[55] ATLAS Collaboration Collaboration, G. Aad et al., "Measurement of the charge asymmetry in top quark pair production in pp collisions at sqrt(s) $=7 \mathrm{TeV}$ using the ATLAS detector," Eur.Phys.J. C72 (2012) 2039, arXiv:1203.4211 [hep-ex].

[56] G. Rodrigo, "The ttbar asymmetry in the Standard Model and beyond," arXiv:1207.0331 [hep-ph].

[57] B. Allanach and Sridhar, "A Supersymmetric Explanation for High $A_{F B}(t \bar{t})$ Via R-Parity Violation," arXiv:1205.5170 [hep-ph].

[58] K. Hagiwara and J. Nakamura, "Diquark contributions to Top quark charge asymmetry at the Tevatron and LHC," arXiv:1205.5005 [hep-ph]. 
[59] D. Chang and W.-Y. Keung, "New limits on R-parity breakings in supersymmetric standard models," Phys.Lett. B389 (1996) 294-298, arXiv:hep-ph/9608313 [hep-ph].

[60] J. M. Cline and S. Raby, "Gravitino induced baryogenesis: A Problem made a virtue," Phys.Rev. D43 (1991) 1781-1787.

[61] M. I. Gresham, I.-W. Kim, S. Tulin, and K. M. Zurek, "Confronting Top AFB with Parity Violation Constraints," arXiv:1203.1320 [hep-ph]. 4 pages.

[62] A. Arhrib, R. Benbrik, and C.-H. Chen, "Forward-backward asymmetry of top quark in diquark models," Phys.Rev. D82 (2010) 034034, arXiv:0911.4875 [hep-ph].

[63] A. V. Manohar and M. B. Wise, "Flavor changing neutral currents, an extended scalar sector, and the Higgs production rate at the CERN LHC," Phys.Rev. D74 (2006) 035009, arXiv:hep-ph/0606172 [hep-ph].

[64] M. Bouchiat and C. Bouchiat, "Parity violation in atoms," Rept.Prog.Phys. 60 (1997) 1351-1396.

[65] K. Nakamura et al. J. Phys G. G 37 (2010) 075021.

[66] S. Porsev, K. Beloy, and A. Derevianko, "Precision determination of electroweak coupling from atomic parity violation and implications for particle physics," Phys.Rev.Lett. 102 (2009) 181601, arXiv:0902.0335 [hep-ph].

[67] S. Porsev, K. Beloy, and A. Derevianko, "Precision determination of weak charge of ${ }^{133}$ Cs from atomic parity violation," Phys.Rev. D82 (2010) 036008, arXiv:1006.4193 [hep-ph].

[68] R. Carlini, "The Qweak experiment: A precision measurement of the proton's weak charge," AIP Conf.Proc. 1261 (2010) 172-178.

[69] R. D. Young, R. D. Carlini, A. W. Thomas, and J. Roche, "Testing the standard model by precision measurement of the weak charges of quarks," Phys.Rev.Lett. 99 (2007) 122003, arXiv:0704.2618 [hep-ph].

[70] CDF Collaboration Collaboration, T. Aaltonen et al., "Measurement of the Top Quark Mass in the All-Hadronic Mode at CDF," arXiv:1112.4891 [hep-ex].

[71] ATLAS Collaboration Collaboration, G. Aad et al., "Measurements of top quark pair relative differential cross-sections with ATLAS in pp collisions at sqrt(s) $=7$ TeV," arXiv:1207.5644 [hep-ex].

[72] A. V. Manohar and M. Trott, "Electroweak Sudakov Corrections and the Top Quark Forward-Backward Asymmetry," arXiv:1201.3926 [hep-ph]. 5 pages, 2 figures. 
[73] J. Aguilar-Saavedra and M. Perez-Victoria, "Probing the Tevatron t tbar asymmetry at LHC," JHEP 1105 (2011) 034, arXiv:1103.2765 [hep-ph].

[74] UTfit Collaboration Collaboration, M. Bona et al., "Model-independent constraints on $\Delta \mathrm{F}=2$ operators and the scale of new physics," JHEP 0803 (2008) 049, arXiv:0707.0636 [hep-ph].

[75] S. Dimopoulos and G. Giudice, "Naturalness constraints in supersymmetric theories with nonuniversal soft terms," Phys.Lett. B357 (1995) 573-578, arXiv:hep-ph/9507282 [hep-ph].

[76] A. G. Cohen, D. Kaplan, and A. Nelson, "The More minimal supersymmetric standard model," Phys.Lett. B388 (1996) 588-598, arXiv:hep-ph/9607394 [hep-ph].

[77] R. Barbieri, E. Bertuzzo, M. Farina, P. Lodone, and D. Pappadopulo, "A Non Standard Supersymmetric Spectrum," JHEP 1008 (2010) 024, arXiv:1004.2256 [hep-ph].

[78] N. Craig, D. Green, and A. Katz, "(De)Constructing a Natural and Flavorful Supersymmetric Standard Model," JHEP 1107 (2011) 045, arXiv:1103.3708 [hep-ph].

[79] C. Brust, A. Katz, and R. Sundrum, "SUSY Stops at a Bump," JHEP 1208 (2012) 059, arXiv:1206.2353 [hep-ph].

[80] C. Brust, A. Katz, S. Lawrence, and R. Sundrum, "SUSY, the Third Generation and the LHC," JHEP 1203 (2012) 103, arXiv:1110.6670 [hep-ph].

[81] J. Cao, Z. Heng, L. Wu, and J. M. Yang, "Top quark forward-backward asymmetry at the Tevatron: A Comparative study in different new physics models," Phys.Rev. D81 (2010) 014016, arXiv:0912.1447 [hep-ph].

[82] J. Goity and M. Sher, "Bounds on delta B = 1 couplings in the supersymmetric standard model," Phys.Lett. B346 (1995) 69-74, arXiv:hep-ph/9412208 [hep-ph].

[83] K. D. Lane and M. Ramana, "Walking technicolor signatures at hadron colliders," Phys.Rev. D44 (1991) 2678-2700. 